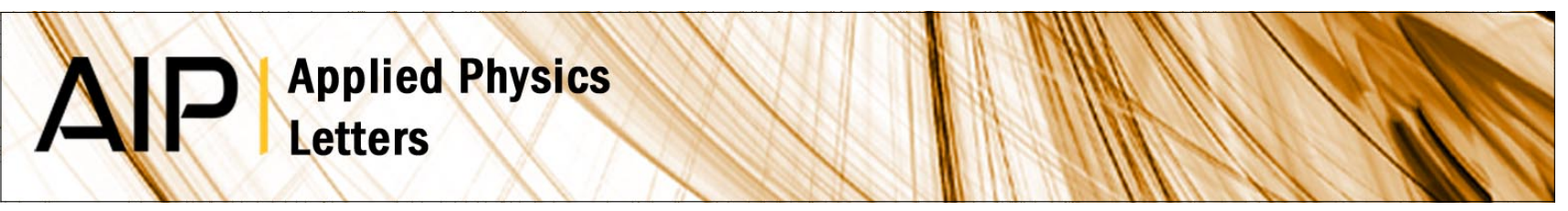

\title{
Wave propagation and tunneling through periodic structures
}

I. V. Konoplev, P. Maclnnes, A. W. Cross, A. D. R. Phelps, L. Fisher et al.

Citation: Appl. Phys. Lett. 93, 231110 (2008); doi: 10.1063/1.3033826

View online: http://dx.doi.org/10.1063/1.3033826

View Table of Contents: http://apl.aip.org/resource/1/APPLAB/v93/i23

Published by the American Institute of Physics.

\section{Related Articles}

Self-induced spin-polarized carrier source in active photonic device with artificial optical chirality Appl. Phys. Lett. 101, 181106 (2012)

Nanocrystalline diamond photonics platform with high quality factor photonic crystal cavities Appl. Phys. Lett. 101, 171115 (2012)

Quasiresonant excitation of $\mathrm{InP} / \mathrm{lnGaP}$ quantum dots using second harmonic generated in a photonic crystal cavity

Appl. Phys. Lett. 101, 161116 (2012)

Enhancement of photocurrent in ultrathin active-layer photodetecting devices with photonic crystals Appl. Phys. Lett. 101, 161103 (2012)

Lithium niobate photonic crystal wire cavity: Realization of a compact electro-optically tunable filter Appl. Phys. Lett. 101, 151117 (2012)

\section{Additional information on Appl. Phys. Lett.}

Journal Homepage: http://apl.aip.org/

Journal Information: http://apl.aip.org/about/about_the_journal

Top downloads: http://apl.aip.org/features/most_downloaded

Information for Authors: http://apl.aip.org/authors

\section{ADVERTISEMENT}
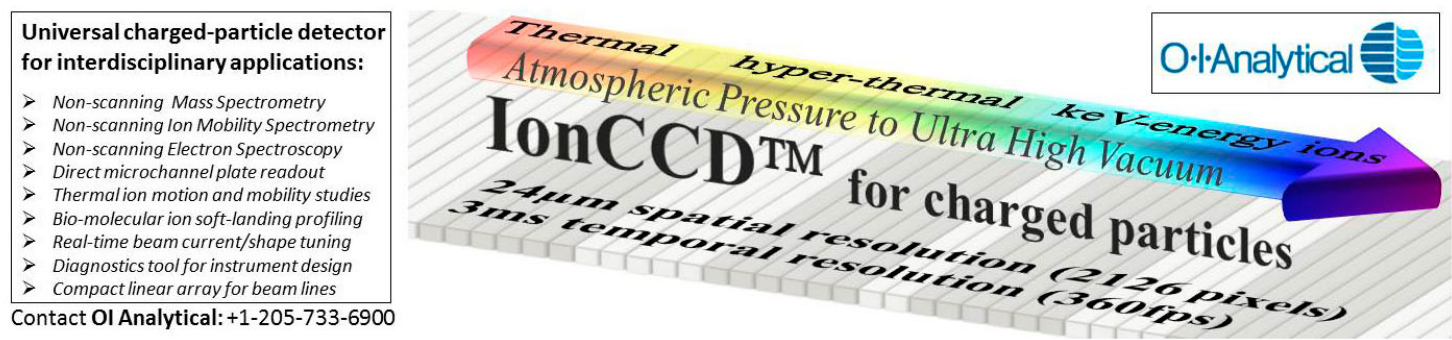


\title{
Wave propagation and tunneling through periodic structures
}

\author{
I. V. Konoplev, ${ }^{\text {a) }}$ P. MacInnes, A. W. Cross, A. D. R. Phelps, L. Fisher, and K. Ronald \\ Department of Physics, SUPA, University of Strathclyde, Glasgow G4 ONG, United Kingdom
}

(Received 2 October 2008; accepted 4 November 2008; published online 10 December 2008)

\begin{abstract}
The phenomenon of tunneling manifests itself in nearly every field of physics. The ability to distinguish a wave tunneling through a barrier from one propagating is important for a number of applications. Here we explore the properties of the wave traveling through the band gap created by a lattice, either as a consequence of tunneling through the barrier or due to the presence of a pass band inside the gap. To observe the pass band for studying tunneling and propagating waves simultaneously, a localized lattice defect was introduced. The differences between the two phenomena are highlighted via waves' dispersion characteristics. (c) 2008 American Institute of Physics. [DOI: 10.1063/1.3033826]
\end{abstract}

The study of properties of a wave tunneling through a band gap is one of the fundamental and divisive problems of physics. ${ }^{1-3}$ The wave tunneling manifests itself in every field of physics including quantum computing, signal processing, optics, electronic, photonics, and condensed matter physics. In this paper we compare the properties of the electromagnetic waves tunneling and propagating through the band gap formed by a one-dimensional (1D) periodic lattice. ${ }^{4}$ In previous works ${ }^{3,5}$ attempts to measure the time required for a single photon or wave packets to tunnel across the 1D photonic band gap have been made and compared with the time required for a photon/wave packet to propagate through the same distance in free space. These studies triggered a number of questions and discussions. ${ }^{1,2,6}$ Here we study the dispersions of the propagated and the tunneled waves to distinguish and compare their properties. We also demonstrate that tunneling and propagating photons can be distinguished via analysis of their dispersion properties.

To conduct the studies a pass band (associated with localized lattice defect eigenmode) inside the band gap was created $^{7,8}$ and the properties of the waves inside and outside the pass band were compared. By creating a periodic lattice with a specific defect it is possible to modify the properties of the lattice band gap and to control the pass band location. ${ }^{8,9}$ As a result, 1D periodic lattices, with and without localized defects, are perfectly suited to study tunneling and propagating phenomena. In this letter, we demonstrate that if the 1D lattice has a finite length $L$, some of the photons having energy and momentum inside the band gap will pass through the barrier gaining a phase shift at the output $\Delta \varphi$ $\equiv \varphi_{\text {out }}-\varphi_{\text {in }}$ proportional to $L$, where $\varphi_{\text {in,out }}$ are the photons' phases at the input and output of the structure. We show that an electromagnetic wave at the exact Bragg resonance has a phase change at the output of the structure equal to 0 , i.e., $\Delta \varphi=0$, which makes the waves at the input and output of the structure "indistinguishable," i.e., changing the phase of the photon at one side of barrier will result in the same change in the photon phase at another end of the barrier. We also show that a localized defect inside a periodic structure significantly alters the lattice dispersive properties. It is demonstrated that the gradient of the wave dispersion inside the pass band is

\footnotetext{
${ }^{\text {a) }}$ Author to whom correspondence should be addressed. Electronic mail: ivan.konoplev@strath.ac.uk. Tel.: +44-141-5484272. FAX: +44-1415522891 .
}

the same as for a propagating wave in a conventional waveguide and significantly differs from one outside the pass band. The gradient difference observed was constant and did not depend on either the defect or the lattice parameters. This allows us to distinguish a "tunneled" wave from a wave that propagates through the barrier due to a lattice defect. We have also observed that a photon located at the pass band center gains zero phase change while passing through the barrier. The results observed are important for determining the lattice suitability for a number of applications including narrow band filters, mirrors, and specialized wave guiding. Also in spite of the fact that the system studied is a $1 \mathrm{D}$ periodic structure based on a cylindrical waveguide, the results observed are applicable for any particle/wave traveling or tunneling through a band gap.

The band gap formation due to the interaction between an electromagnetic wave and a 1D periodic structure takes place in the vicinity of the Bragg resonance defined as $\vec{\delta}$ $=\overrightarrow{\bar{k}}-\vec{k}_{i}+\vec{k}_{s}$, where $\vec{k}_{i, s}$ are the wave vectors of the incident and scattered waves and $\left|\vec{k}_{i, s}\right|=2 \pi f / v, v$ is the speed of light in the unperturbed medium and $f$ is the wave frequency, $\vec{k}$ is the lattice eigenvector $|\vec{k}|=2 \pi / d$, $d$ is the lattice period, and $\vec{\delta}$ is the detuning from the resonance condition $\vec{k}=\vec{k}_{i}-\vec{k}_{s}$. In this letter, a low contrast 1D periodic lattice based on dielectric inserts, having a periodic refractive index [the variation in the refractive index $\Delta n$ is small in comparison with the refractive index of the unperturbed dielectric $\left.n_{0}\left(\Delta n / n_{0} \ll 1\right)\right]$, inside a smooth cylindrical metal waveguide was studied (Fig. 1). ${ }^{8}$ The annular inserts had inner and outer radii of 5.0 and $11.0 \mathrm{~mm}$, respectively, with an unperturbed refractive index of $\sim 1.87$. The periodicity of the refractive index of the dielectric structure was achieved via a corrugation of the inner surface of the inserts (the period of corrugation is 10.0

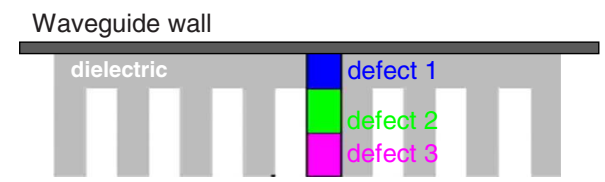

FIG. 1. (Color online) Schematic of the 1D periodic structures (side view) with corrugated dielectric insert (the air, dielectric, and waveguide walls are shown by the white, gray, and black colors) with localized "zero" length defects of the period. Defect 1 has a radius equal to the maximum radius of the corrugation; defect 2 has an intermediate radius; and defect 3 has a radius equal to the minimum corrugation radius. 

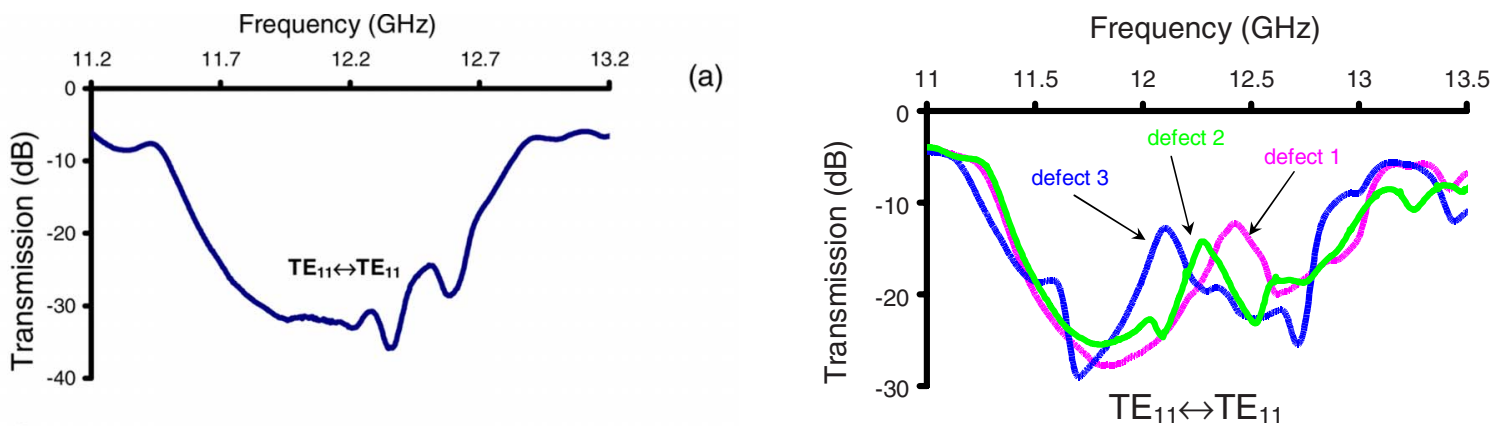

(a)

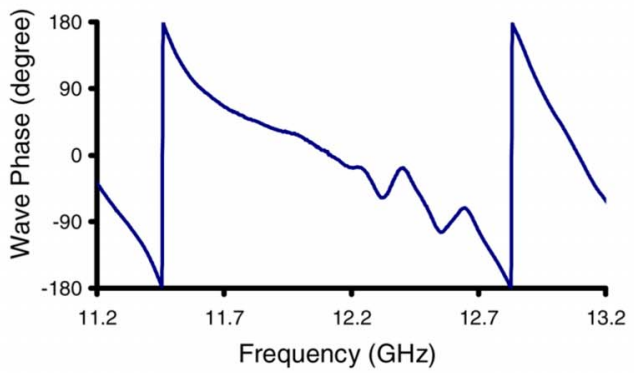

(b)

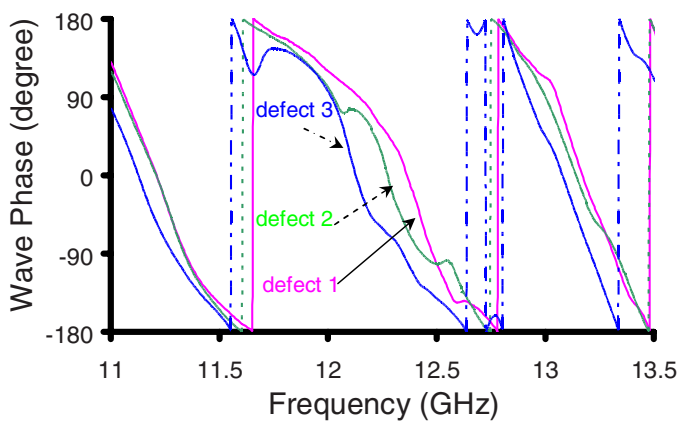

(b)

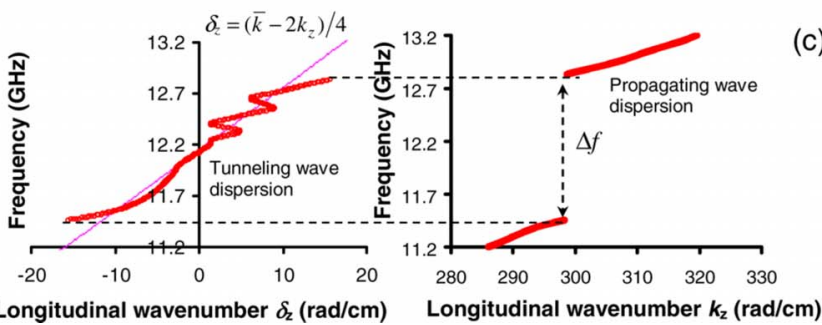

FIG. 3. (Color online) Results of the experimental studies of transmission through the 1D Bragg dielectric structures with zero length defects. (a) Dependence of the transmission through the structures with the defects. (b) Measured wave's phase variation with the frequency at the output of the structures.

FIG. 2. (Color online) Experimental study of the regular 1D periodic structure. (a) Dependence of the transmission profile vs frequency. (b) Variation in the wave's phase at the output of the structure vs wave frequency. (c) The dispersion diagrams of the periodic structure in wave number ranges $[-20 ; 20]$ (left diagram) and $[280,330]$ (right diagram) evaluated from experimental data (bold line) and calculated (thin line).

$\mathrm{mm}$ and the corrugation depth is $4.0 \mathrm{~mm}$ ). In the case of the periodic structure, with a lattice defect, the defect was made by inserting a dielectric ring between two regular periodic structures of equal length (Fig. 1). Figure 1 illustrates three periodic structures with localized defects having different refractive indices and used in the experiments. The experiments were carried out using a vector network analyzer capable of both wave amplitude and phase measurements. At the start the experimental apparatus was calibrated to reduce the background noise signal and to measure the relative wave's phase at the output of the lattice, i.e., to measure the phase difference between input and output waves. To conduct the experiments the fundamental $\mathrm{TE}_{11}$ mode (the photons having the smallest transverse momentum allowed in such a structure) of the cylindrical waveguide was used. The frequency-chirped electromagnetic wave was launched and the amplitude (transmission coefficient) and the phase of the signal at the output of the structure were measured in the frequency range from 11.0 to $13.5 \mathrm{GHz}$. In this frequency range the formation of the band gap (barrier) associated with $\mathrm{TE}_{11} \leftrightarrow \mathrm{TE}_{11}(11.5-12.8 \mathrm{GHz})$ wave scattering takes place. ${ }^{8}$ In this case the expression for the detuning $\delta_{z}\left(\delta_{z}\right.$ is the projection of $\vec{\delta}$ on the longitudinal axis z) can be simplified $\delta_{z}=\bar{k}-2 k_{z}$, where $k_{z}=k_{s z, i z}=\sqrt{(2 \pi f / v)^{2}-k_{s \perp, i \perp}^{2}}$ and $k_{s \perp, i \perp}$ are the transverse wave numbers of the scattered and incident waves, respectively. In the first set of experiments a conventional 1D Bragg structure was investigated. In Figs. 2(a) and 2(b) the results observed from the measurement of the

wave's transmission and phase versus frequency are shown. The phase data observed [Fig. 2(b)] were analyzed and the dispersion characteristic of the periodic structure was obtained [Fig. 2(c)]. The dispersion diagrams have been evaluated from phase data due to the direct link between the longitudinal wave number $k_{z}$ and the phase of the transmitted signal $\left(\varphi=k_{z} L\right.$, where $L$ is the length of the structure $\left.{ }^{10}\right)$. To obtain the $\left(f ; \delta_{z}\right)$ diagram (dispersion inside the band gap region), the Bragg resonance condition was taken into account. The band gap observed in Fig. 2(c) is indicated as $\Delta f$ on the right hand side diagram and agreed well with the profile of the transmission coefficient in Fig. 2(a). The left hand figure shows the wave dispersion inside the frequency interval associated with the band gap (11.5-12.8 GHz). The straight line on the left graph (best fit line to the measured dispersion) is obtained using the following expression: $\delta_{z}$ $\approx\left(\bar{k}-2 k_{z}\right) / g$, where $g$ is a constant approximately equal to 4 . Let us note that the dispersion relation for the propagating wave should be obtained from the definition of the detuning, i.e., $\delta_{z} \approx\left(\bar{k}-2 k_{z}\right)$, and it is clear that the gradient of the dispersion inside the band gap frequency range is larger as compared with the dispersion outside the band gap [see also the phase variation in Fig. 2(b)]. The gradient observed in the experiments was the same for different lattice parameters, i.e., parameter $g \sim 4$ observed here empirically was constant for all experiments. It is possible to assume that this parameter depends on material properties, for instance, a refractive index; however more experiments will be required to identify this dependence. One also notes that the only wave that tunneled through the band gap at the exact Bragg frequency gained no phase change. Therefore the wave phase velocity observed inside the band gap is large as compared with the phase velocity of the propagating wave but still has a finite value except for a single point associated with the exact 

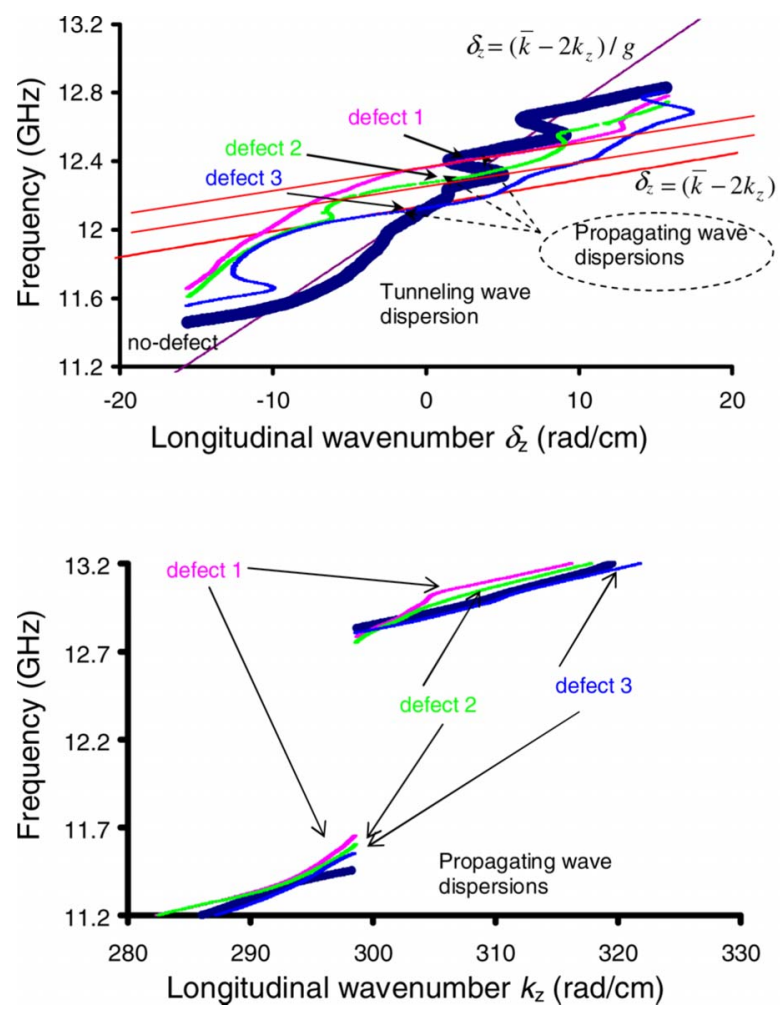

FIG. 4. (Color online) The comparison of the dispersion diagrams of the periodic structures with defects (thin lines) and without (bold lines) in the wave number ranges (a) $[-20 ; 20]$ and (b) $[280,330]$. The straight red lines in (a) indicate the gradient of the dispersions associated with waves propagating through the band gap due to the presence of the pass band and the brown line indicates the gradient of the dispersion associated with the tunneled wave.

Bragg frequency. This indicates a change in the wave's properties during the tunneling through such a barrier unless it has a specific Bragg frequency defined by the lattice.

To compare the properties of the propagating and tunneling photons, a zero length defect inside the $1 \mathrm{D}$ periodic structure has been introduced and its influence on the band gap parameters has been studied in the $11.0-13.5 \mathrm{GHz}$ frequency range. In Fig. 3 the transmission coefficients versus frequency [Fig. 3(a)] as well as the results of the phase measurements [Fig. 3(b)] are shown. The transmission profiles and the frequency dependence of the phase illustrate the presence of pass bands associated with the defect, as well as strong dependence of the pass band location on the value of the defect's refractive index. The variation in the defect refractive index results in a frequency shift in the pass band position [Fig. 3(a)] and alteration of the waves' dispersions [Fig. 4(a)]. Although the lines of the dispersions associated with the pass bands are frequency shifted, it is clear that the dispersions are parallel to each other [Fig. 4(a)]. Analyzing the data the difference between the waves propagating through the pass band and the waves tunneling through the barrier [Fig. 4(a) compared with Fig. 2(c)] can be observed. Indeed, the gradients of the dispersion characteristics [Fig. 4(a)] outside the pass bands coincide well with the gradient of the dispersion of the lattice without the defect [Fig. 2(c)], while the gradients of the dispersions inside the pass band (parallel and frequency shifted lines) are the same as the gradient of the dispersion of the wave propagating in a smooth waveguide. Let us note that to obtain the straight lines [Fig. 4(a)], which fit with the dispersion inside the pass band, the expression $\delta_{z}=\left(\bar{k}-2 k_{z}\right)$ was used. In contrast $\delta_{z}$ $=\left(\bar{k}-2 k_{z}\right) / g$, where $g$ is a constant approximately equal to 4 , was used to observe the line that either fits with the dispersion inside the band gap [Fig. 2(c)] or is parallel (having the same gradient) in the case of the lattices with the localized defect [Fig. 4(a)]. Analyzing the dispersion diagrams the boundaries between the pass band and the band gap as well as the center of the pass band can be defined.

To conclude, 1D band gap structures were investigated, with the results of the experimental studies presented and discussed. We demonstrate that the dispersion of the wave tunneling through the barrier is different from the dispersion of the wave propagating through the lattice due to a pass band, allowing us to distinguish two different phenomena. It is demonstrated that the gradient of the dispersions associated with the wave that have tunneled through the band gap is larger as compared with the gradient of the dispersions associated with the wave propagating through the lattice due to the presence of the pass band and can be used to differentiate the waves. The analysis of the dispersion characteristics allows one to define the boundaries of the band gaps and pass bands as well as the position of the defect eigenmodes (pass band center). It is demonstrated that the dispersion diagrams provide information about lattice structure and can be used to identify the characteristics of lattice defects such as refractive index via analysis of pass band location, which strongly depends on the variation in the defect refractive index. We have to stress that we did not demonstrate superluminal behavior of wave tunneling through the band gap as the dispersion's gradient in this case cannot be considered as an energy propagation velocity.

The authors thank EPSRC, U.K., for their support.

${ }^{1}$ M. Büttiker and S. Washburn, Nature (London) 422, 271 (2003).

${ }^{2}$ H. G. Winful, Proc. SPIE 6664, 66640C (2007).

${ }^{3}$ S. Doiron, A. Hache, and H. G. Winful, Phys. Rev. A 76, 023823 (2007). ${ }^{4}$ H. Kogelnik and C. V. Shank J. Appl. Phys. 43, 2327 (1972); D. C. Flanders, H. Kogelnik, R. V. Schmidt, and C. V. Shank, Appl. Phys. Lett. 24, 194 (1974); H. G. Winful, ibid. 46, 527 (1985).

${ }^{5}$ A. M. Steinberg, P. G. Kwait, and R. Y. Chiao, Phys. Rev. Lett. 71, 708 (1993); T. E. Hartman, J. Appl. Phys. 33, 3427 (1962); M. PalominoOvando, H. A. Coyotecatl, R. Esquivel-Sirvent, and G. H. Cocoletzi, Phys. Lett. A 267, 429 (2000); Ch. Spielmann, R. Szipöcs, A. Stingl, and F. Krausz, Phys. Rev. Lett. 73, 2308 (1994); A. B. Shvartsburg, M. Marklund, G. Borodin, and L. Stenflo, Phys. Rev. E 78, 016601 (2008).

${ }^{6}$ H. G. Winful, Phys. Rev. A 76, 057803 (2007).

${ }^{7}$ S.-H. Kim and C. G. Fonstad, IEEE J. Quantum Electron. QE-15, 1405 (1979); R. D. Meade, K. D. Brommer, A. M. Rappe, and J. D. Joannopoulos, Phys. Rev. B 44, 13772 (1991); L. I. Deych, A. Yamilov, and A. A. Lisyansky, Europhys. Lett. 46, 524 (1999).

${ }^{8}$ I. V. Konoplev, P. McGrane, A. D. R. Phelps, A. W. Cross, and K. Ronald, Appl. Phys. Lett. 87, 121104 (2005); I. V. Konoplev, P. McGrane, A. W. Cross, K. Ronald, and A. D. R. Phelps, J. Appl. Phys. 97, 073101 (2005); I. V. Konoplev, G. Doherty, A. W. Cross, A. D. R. Phelps, and K. Ronald, Appl. Phys. Lett. 88, 111108 (2006); I. V. Konoplev, P. MacInnes, A. W. Cross, A. D. R. Phelps, and K. Ronald, ibid. 91, 171107 (2007).

${ }^{9}$ E. Yablonovitch, Phys. Rev. Lett. 58, 2059 (1987); S. Lan, S. Nishikawa, Y. Sugimoto, N. Ikeda, K. Asakawa, and H. Ishikawa, Phys. Rev. B 65, 165208 (2002); V. I. Kopp and A. Z. Genack, Phys. Rev. Lett. 89, 033901 (2002); O. Painter, R. K. Lee, A. Scherer, A. Yariv, J. D. O’Brien, P. D. Dapkus, and I. Kim, Science 284, 1819 (1999); O. Cohen, B. Freedman, J. W. Fleischer, M. Segev, and D. N. Christodoulides, Phys. Rev. Lett. 93, 103902 (2004).

${ }^{10}$ G. Burt, S. V. Samsonov, K. Ronald, G. G. Denisov, A. R. Young, V. L. Bratman, A. D. R. Phelps, A. W. Cross, I. V. Konoplev, W. He, J. Thomson, and C. G. Whyte, Phys. Rev. E 70, 046402 (2004). 